\title{
A Novel Hybrid Segmentation Method with Particle Swarm Optimization and Fuzzy C-Mean Based On Partitioning the Image for Detecting Lung Cancer
}

\author{
P Kavitha, S Prabakaran
}

\begin{abstract}
:
Recently, the medical image processing is extensively used in several areas. In earlier detection and treatment of these diseases is very helpful to find out the abnormality issues in that image. Here there are number of methods available for segmentation to detect the lung nodule of computer tomography (CT) image. The main result of this paper, the earlier detection of lung nodules using Pre-processing techniques of top-hat transform, median and adaptive bilateral filter was compared both filtering methods and proved the adaptive bilateral filter is suitable method for CT images. The proposed segmentation technique uses novel strip method and the image is split into number of strips 3, 4, 5 and 6. A marker-watershed method based on PSO and Fuzzy C-mean Clustering method was proposed method. Firstly, the input image was reduced noise reduction and smoothing and the filter image is using strips method and then the image is segmented by marker watershed method. Secondly, the enhanced PSO technique was used to locate the better accurate value of the clustering centers of Fuzzy C-mean Clustering. Final stage, with the accurate value of centers and the enhanced target function and the small region of the segmented object was clustered by Fuzzy C-mean. In segmentation algorithm presented in this paper gives $95 \%$ of accuracy rate to detect lung nodules when strip count is 5 .
\end{abstract}

Index Terms: Adaptive bilateral, Marker Watershed, PSO, Fuzzy C-mean, GLCM, SVM

\section{INTRODUCTION}

Recently the medical image techniques are most important technology is screening of cancer images. Computer Tomography is a standard modality of detecting and assessing cancer images. The nodules are differentiate benign and malignant. For the present study, pre-processing of original sample image to reduce noise detection and gaussian blur using adaptive bilateral filter, by comparing adaptive bilateral filter is best method to find better accurate rate. Three segmentation techniques are compared and proved the combination of marker based watershed algorithm based on PSO and Fuzzy C-mean was proved best accuracy value measured using the True positive, True negative, False

P.Kavitha, Dept. of Information Technology, Bharath Institute of Higher Education and Research, Channai, India.

S.Prabakaran, Computer Science and Engineering, SRM University, Kattankulathur, India. positive and False negative values. It was proved that the combination of adaptive bilateral filter and Marker watershed based on PSO and FCM algorithm has more accurate results among others.

\section{LiteratURE REVIEW}

Emre Dandl [1] was proposed method to classify the lung nodules. In LUVEM (Lung Volume Extraction Method), using median filter to reduce the noise and to implement LUVEM method to extract the nodules from CT scan image. After that the Self Organizing Method (SOM) is successful detection of early stages. It's unsupervised neural network method and easily segmented to small nodules on the lung nodules. In feature extraction, first we used shape based method for analyzing lung nodule image and to utilize GLCM method is used to statistic of gray level lung nodule. Finally the lung nodules are extracted and computed to the segmentation object. Finally, using Probabilistic Neural Network (PNN) to classify the bengin and malign lung nodule. The result $f$ the proposed classification method of PNN is given better accuracy value.

Lavanya M, Muthu Kannan P was proposed comparative analysis of FCM and FLICM algorithm. Pre-processing the input image to reduce noise reduction and histogram equalization is done using median filer and filtering image is using segmentation of Modified Expectation Maximization (MEM) algorithm. This algorithm to initialize the parameter of expectation and maximization and the value is increased step by step. Finally, calculate the new maximization and expectation position. After that the completion of segmentation to extract the nodule is utilized for feature extraction method such area, perimeter and eccentricity. Final steps to classification method to compared better best performance measures of FCM and FLICM. The performance measures of specificity $92 \%$, precision $97 \%$, sensitivity $82 \%$ and accuracy $95 \%$ [2].

The author is mainly focused to implement soft computing methods. In pre-processing, various filtering methods median, gabor filter and anisotropic filtering are compared and proved anisotropic filter are more suitable for pre-processing. 


\section{A Novel Hybrid Segmentation Method with Particle Swarm Optimization and Fuzzy C-Mean Based On Partitioning the Image for Detecting Lung Cancer}

A Marker based algorithm is used to segment the lung nodule to extract the image. Finally, compared SVM and KNN classification result the KNN classifier to provide better accuracy value of the image and this proposed method was implemented by Shraddha G. Kulkarni, Sahebrao B. Bagal [3].

\section{Methodology}

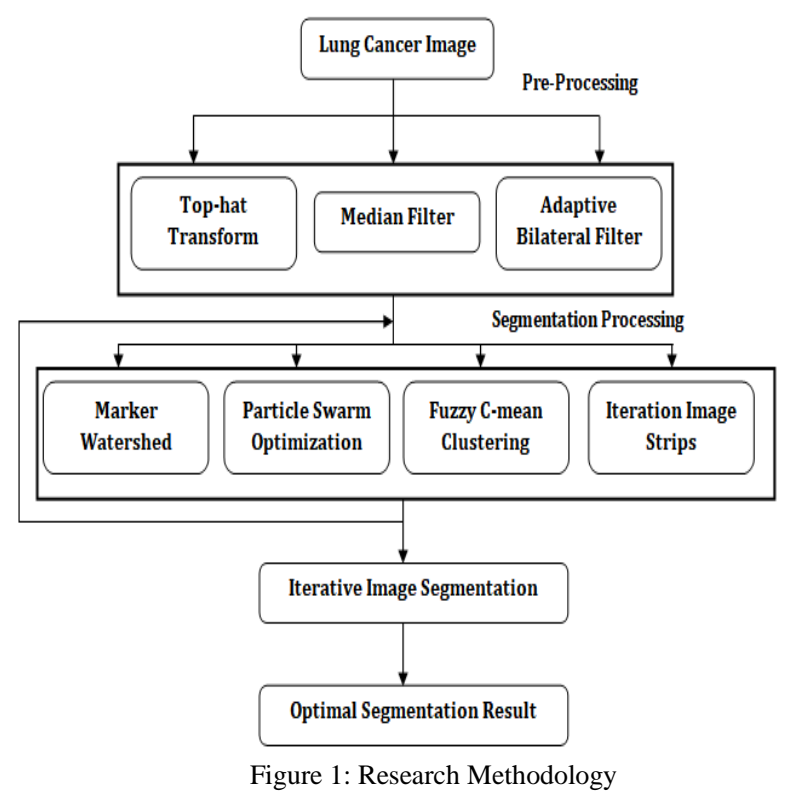

\section{A. Pre-processing}

In pre-processing, the input lung CT image is being processed to enhanced image quality. The various pre-processing technique is used to reduced noise effect and blurring effect. The better quality measurements are depends on the various methods.

\section{B. Filtering}

Pre-processing techniques of top-hat transform, median and adaptive bilateral filter was compared and the proposed method adaptive bilateral is filter is suitable for pre-processing to $\mathrm{CT}$ image.

\section{Top-hat transform}

The top-hat transform technique in morphology operation and image processing. In this method, the elements are extracted from original image according to small or narrow features, bright or dark features. It works by computing morphological operation in openings is subtracted for the original image [4]. The filtering function defined as:

$$
\begin{gathered}
\mathrm{SE}=\text { strel }(\text { 'disk', r) } \\
\text { Input=imtophat }(\mathrm{I}, \mathrm{SE})
\end{gathered}
$$

\section{Median Filter}

In this method, to remove salt and pepper noise of original image and retains the sharpness of the image. Each pixel is replaced by the median value from the neighbourhood pixels and a window size $3 \times 3$ [5].

\section{Adaptive Bilateral Filter}

Original image is converted to gray scale image. Bilateral filter is a non-liner, edge preserving and reducing noise for images. It replaces of each pixel intensity with a weighted average values from neighbourhood pixel. The weight average of pixel value is based on the gaussian blur. Its defined as:

$$
B F[I]_{\mathrm{p}}=\frac{1}{W_{\mathrm{p}}} \sum_{\mathrm{q} \in S} G_{\sigma_{s}}(|\mathrm{p}-\mathrm{q}|) G_{\sigma_{r}}\left(I_{\mathrm{p}}-I_{\mathrm{q}}\right) I_{\mathrm{q}}
$$

Where $\mathrm{W}_{\mathrm{p}}$ is normalization factor:

$$
W_{\mathbf{p}}=\sum_{\mathbf{q} \in \mathcal{S}} G_{\sigma_{s}}(|\mathbf{p}-\mathbf{q}|) G_{\sigma_{r}}\left(I_{\mathbf{p}}-I_{\mathbf{q}}\right)
$$

Where,

$\sigma_{\mathrm{s}}$ and $\sigma_{\mathrm{r}}$ - compute quantity of filtering input image

$\mathrm{G}_{\sigma \mathrm{s}}-$ To decrease distant pixels

$\mathrm{G}_{\sigma \mathrm{\sigma r}}$ - If decrease a gaussian the influence of pixels $q$ with intensity value different from $\mathrm{I}_{\mathrm{p}}$.

When tuning the parameter of $\sigma_{\mathrm{r}}$ increases, the filter is very closer to gaussian blur because the range gaussian is flatter and the parameter $\sigma_{\mathrm{s}}$ increases, smoothening larger features

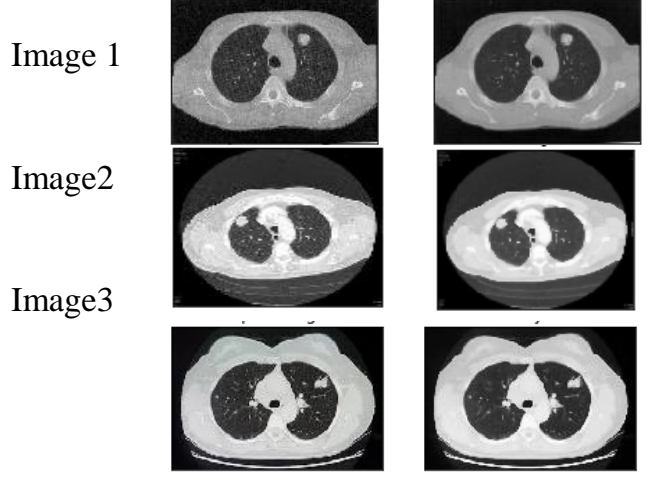

Figure 2: (a) Original Image (b) Adaptive Bilateral Filter

Table 1: The input image values of MSE and PSNR

\begin{tabular}{|c|c|c|c|}
\hline \multicolumn{5}{|c|}{ MSE } \\
\hline $\begin{array}{c}\text { Sample } \\
\text { Image }\end{array}$ & $\begin{array}{c}\text { top-hat } \\
\text { transform }\end{array}$ & $\begin{array}{c}\text { Median } \\
\text { Filter }\end{array}$ & $\begin{array}{c}\text { Adaptive } \\
\text { Bilateral Filter }\end{array}$ \\
\hline Img1 & 79.218 & 64.08 & 62.377 \\
\hline Img2 & 80.135 & 68.374 & 67.319 \\
\hline Img3 & 83.724 & 61.192 & 59.427 \\
\hline Img4 & 80.239 & 66.135 & 65.194 \\
\hline Img5 & 82.192 & 70.493 & 69.196 \\
\hline
\end{tabular}

\begin{tabular}{|c|c|c|c|}
\hline \multicolumn{4}{|c|}{ PSNR } \\
\hline Sample Image & $\begin{array}{c}\text { top-hat } \\
\text { transform }\end{array}$ & $\begin{array}{c}\text { Median } \\
\text { Filter }\end{array}$ & $\begin{array}{c}\text { Adaptive } \\
\text { Bilateral } \\
\text { Filter }\end{array}$ \\
\hline Img1 & 79.722 & 85.483 & 87.217 \\
\hline Img2 & 76.386 & 80.291 & 81.394 \\
\hline Img3 & 78.331 & 82.492 & 82.971 \\
\hline Img4 & 77.487 & 85.742 & 87.485 \\
\hline Img5 & 79.381 & 84.497 & 85.941 \\
\hline
\end{tabular}

In this filter technique, the adaptive bilateral filter gives more accurate rate of the original image. 


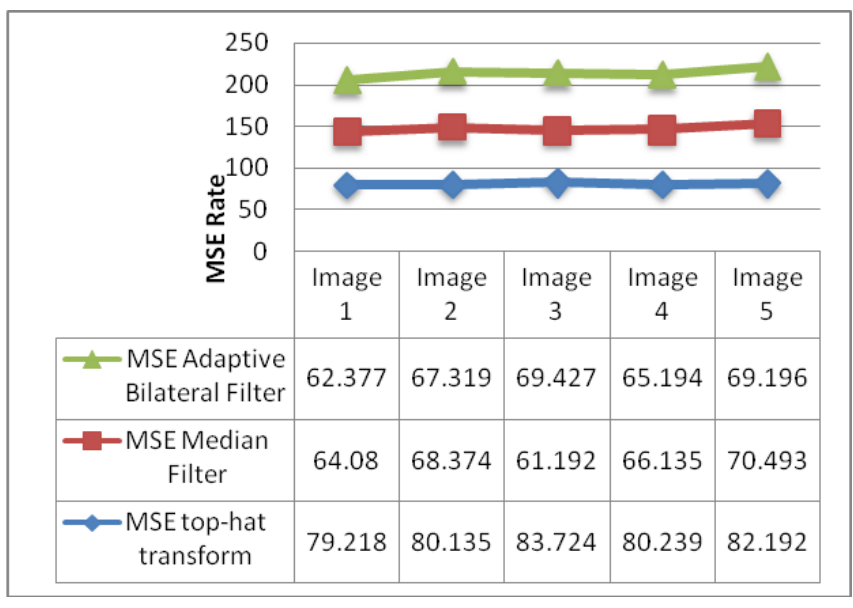

Figure 3: Comparative results of MSE

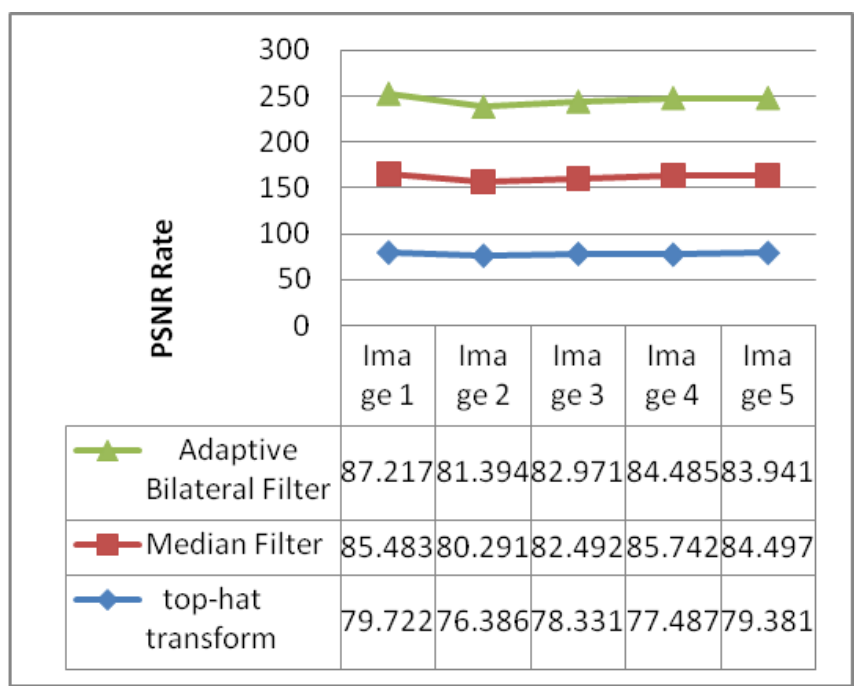

Figure 4: Comparative results of PSNR

\section{A. Segmentation}

In image processing, the segmentation techniques are used to partition a medical image into multiple segments. It's going to find the image locate an object and boundaries like a lines, curves, etc. In this proposed method, the filter image is subdivided into five horizontal equidistance strips. The strips are numbered str1, str2, str3, str4 and str5.

The strip str 2 is considered for segmentation
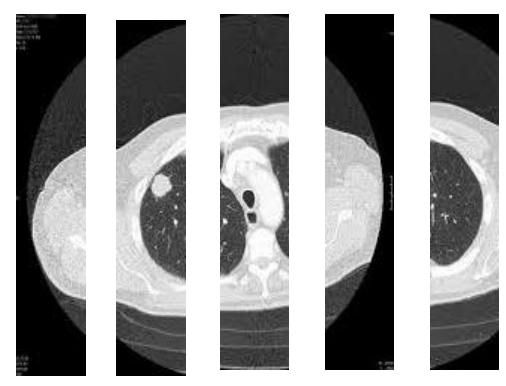

Figure 5: Filter image subdivided into five horizontal equidistance strips

\section{Marker based watershed transform}

In this proposed method, the image strip str2 is binarized using Otsu's thresholding method to perform graythresh() function and the image is converted to binary format. A structuring element is defined based on the original image. Its computing foreground and background markers. Final stage, the watershed transform image is executed.

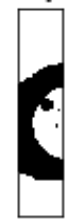

(a)

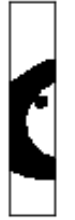

(b)

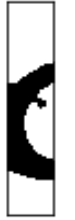

(c)
Figure 6: (a) Otsu's Thresholding (b) Morphology Operation Closing (c) Dilation

\section{Fuzzy C-mean based on Particle Swarm Optimization}

Traditionally, the fuzzy c-mean clustering method without consideration of neighbour information and the efficiency is low rate [6]. In these proposed techniques, the clustering center are initialized in the particle swarm optimization (PSO) and the traditional fuzzy c-mean clustering is also improved. The algorithm is defined (1) Cluster and Iterations are initializing (2) Parameter

Particle Swarm Optimization (PSO)

The PSO algorithm is given as:

Step1: Cluster and Iterations are initializing

Step2: Parameter p, sc, fc, numsucc $=0$, and numfail $=0$ are initializing

Step3: Identify a fitness function

Step4: To find the fitness of each particle rate

Step5: Update the local best solution.

Step6: Steps 4 and 5 repeat

Step7: Each particle velocity and position are updated

Step8: Execute the selection operator.

Step9: If any local best position $y_{i}$ has changed, and to perform the clustering algorithm.

Step10: End procedure

In this method focuses on the current best position of a new particle, the new particle is considered as the swarm and the velocity update equation for new particle is defined as:

$v \varphi(t+1)=x \varphi(t)+\operatorname{pbest}(t)+\omega v \varphi(t)+\rho(t)(1-2 r)$.

The gbest position is to improve the random search area around the position. The $r$ and $p(t)$ is random vector and diameter of the search area. The range of the random vector lies between0and 1 .

The diameter of the search area can be updated using the following equation:

$$
\rho(t+1)= \begin{cases}2 \rho(t), & \text { \#successes }>\mathrm{sc}, \\ \left(\frac{1}{1.5}\right) \rho(t), & \text { \#failures }>\mathrm{fc}, \\ \rho(t), & \text { otherwise, }\end{cases}
$$

Where, 


\section{A Novel Hybrid Segmentation Method with Particle Swarm Optimization and Fuzzy C-Mean Based On Partitioning the Image for Detecting Lung Cancer}

sc and $\mathrm{fc}$ - Threshold parameters, the value of $\mathrm{sc}=15$ and $\mathrm{fc}=5$

\section{Enhanced Fuzzy C-mean}

Enhanced FCM method, the relativity between the gray value of small region and single pixel value is not considered. So a new concept $h_{i}$ called gray dissimilarity is proposed method. $h_{i}=$ Average (i)- $x_{k}$ means the gray dissimilarity between the average gray value and pixel value $x_{k}$. In this equation for calculating distance from the pixel $\mathrm{x}_{\mathrm{k}}$ to the clustering center is also redefined as $\mathrm{d}_{\mathrm{ik}}=\mathrm{h}(\mathrm{i})\left\|\mathrm{x}_{\mathrm{k}}-\mathrm{vi}\right\|^{2}$

The improved clustering function

$$
J(U, V)=\sum_{k=1}^{n} \sum_{i=1}^{c} \mu_{i k}^{m} h(i)\left\|x_{k}-v_{i}\right\|^{2}
$$

In this equation, the variation between the typical gray value of the $i^{\text {th }}$ is a small region and the pixel $x_{k}$, the more possibility they belong to the equivalent region, vice versa. The proposed method of new iterating equation of the matrix and clustering center are redefined as:

$$
\mu_{\psi_{k}}^{(+1)}=\frac{1}{\sum_{j=1}^{c}\left(\frac{h(i)}{h(j)} \frac{\left\|x_{2}-v_{i}\right\|}{\left\|x_{k}-v_{j}\right\|}\right)^{i(k-1)}}
$$

The original iterating equation is

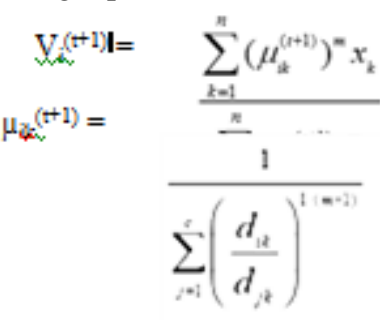

$$
\begin{aligned}
& \mathrm{V}_{i}^{(++1)}=\frac{\sum_{i=1}^{\infty}\left(\mu_{\alpha}^{(j+1)}\right)^{n} x_{i}}{\sum_{i=1}^{\infty}\left(\mu_{\alpha}^{(j+1)}\right)^{n}}
\end{aligned}
$$

The above method to improved clustering function in the data aggregation, clustering center aggregation, weight and matrix aggregation to control result of the clustering function. The proposed method of the segmentation is more accurate compared other methods.

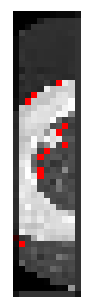

Figure 7: Segmentation of Proposed Method
Table 2: Accuracy measures Proposed Algorithm

\begin{tabular}{|c|c|c|c|c|c|}
\hline $\begin{array}{c}\text { Sample } \\
\text { Image }\end{array}$ & TP & TN & FP & FN & $\begin{array}{c}\text { Accuracy } \\
\text { Rate }\end{array}$ \\
\hline Image 1 & 92.6128 & 99.9999 & 0.0001 & 9.3942 & 96.8179 \\
\hline Image 2 & 88.8404 & 99.9999 & 0.0001 & 11.1592 & 94.4204 \\
\hline Image 3 & 87.2946 & 99.9999 & 0.0001 & 12.7054 & 93.6473 \\
\hline Image 4 & 87.2946 & 99.999 & 0.0001 & 12.7054 & 93.6473 \\
\hline Image 5 & 83.9061 & 99.9999 & 0.0001 & 16.0939 & 91.9531 \\
\hline
\end{tabular}

Table 3: Comparative Results of segmentation algorithm

\begin{tabular}{|c|c|c|c|c|}
\hline $\begin{array}{c}\text { Sample } \\
\text { Image }\end{array}$ & $\begin{array}{c}\text { Marker } \\
\text { Watershed }\end{array}$ & PSO & MWFRM & Proposed \\
\hline Image 1 & 89.3672 & 89.0624 & 88.9813 & 95.8079 \\
\hline Image 2 & 88.5622 & 88.9689 & 86.1018 & 94.4204 \\
\hline Image 3 & 79.8186 & 84.9967 & 83.0334 & 93.6473 \\
\hline Image 4 & 82.4514 & 81.8114 & 83.2876 & 93.6473 \\
\hline Image 5 & 79.9876 & 82.0954 & 81.2033 & 91.9531 \\
\hline
\end{tabular}

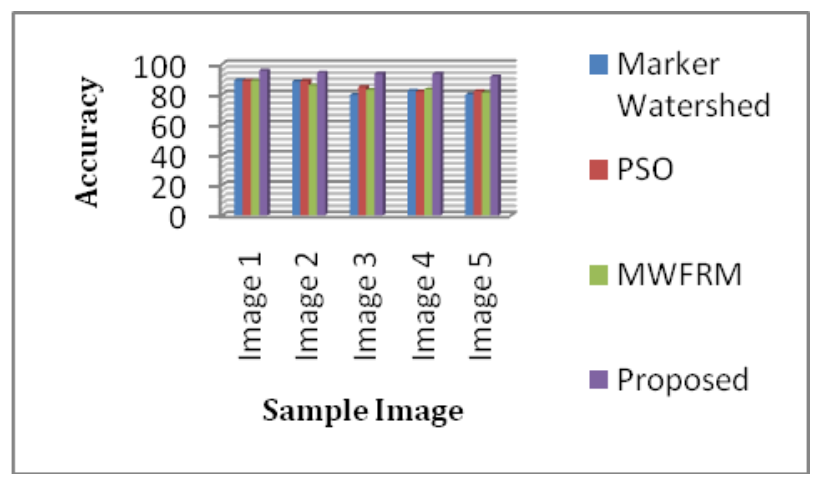

Figure 8: Segmentation of Proposed Method

\section{IMPLEMENTATION}

The above techniques are practically executed by opencv python coding and proposed method results was verified. In the pre-processing, an evaluation of both filter methods was done and measures the performance of top-hat transform filter, median and adaptive bilateral filter.

The MSE and PSNR rate are shown in Table 1 and Figures 2 and 3. From the filtering results, the adaptive bilateral filter has given better accurate rate compared with top-hat transform filter and median filter.

In the proposed method, accuracy was measured using the TP (True Positive), TN (True Negative), FP (False Positive), and FN (False Negative) by comparing the results from the other segmentation algorithm with manual results. 
The results of the proposed segmentation method are shown in Table 2 and graphical view of the segmentation accuracy was calculated using the TP (True Positive), TN (True Negative), FP (False Positive), and FN (False Negative) shown in figure 8. Comparative Results algorithm shown in Table 3

\section{CONCLUSION}

In this paper, different segmentation algorithms have been classified to early detection of lung tumor. The original medical image use to pre-processing the adaptive bilateral filter has given better results by comparing top-hat transform filter and median filter. Analyzing four segmentation techniques, the better accuracy result of the tumor detection is enhanced proposed method with maximum accuracy rate of $95 \%$. The proposed method is more accurate when compared to the existing segmentation algorithm. In future studies, various segmentation algorithm will be integrated and to improve the better accuracy rate.

\section{REFERENCES}

1. Emre Dand11 “A Computer-Aided Pipeline for Automatic Lung Cancer Classification on Computed Tomography Scans" Journal of Healthcare Engineering Volume 2018, Article ID 9409267, 12 pages

2. Lavanya M, Muthu Kannan P "Lung cancer segmentation and diagnosis of lung cancer staging using MEM (modified expectation maximization) algorithm and artificial neural network fuzzy inference system (ANFIS)" ISSN 0970-938X Biomed Research 201829 (14): 2919-2924.

3. Shraddha G. Kulkarni, Sahebrao B. Bagal "Lung Cancer Tumor Detection Using Image Processing And Soft Computing Techniques" International Journal of Science Technology and Management Volume 05 ISSN 2394-1537, May 2016

4. Ada, Rajneet Kaur, "Early Detection and Prediction of Lung Cancer Survival using Neural Network Classifier", IJAIEM volume 2, Issue 6, June 2013

5. G. Gupta, "Algorithm for image processing using improved median filter and comparison of mean, median and improved median filter," International Journal of Soft Computing and Engineering, vol. 1, no. 5, 2011.

6. Gao H, SUI W CH, HOU CH H. Improved techniques for automatic image segmentation [J]. IEEE Transactions on Circuits and Systems for Video Technology, 2001, 11(12): 1273-1280. http://dx.doi.org/10.1109/76.974681

7. Neelam Marshkole, Bikesh Kumar Singh,and Thoke, A.S., "Texture and Shape based Classification of Brain Tumors using Linear Vector Quantization" International Journal of Computer Applications, Vol.30, pp.0975 - 8887, 2011 .

8. Moffy Crispin Vas, Prof. Amita Dessai "Classification of Cancerous and Non-Cancerous Lung Cancer Nodules Using Image Processing Techniques" International Journal of Advance Research in Science and Engineering Vol. 6 Issue 4 April 2017

9. P. Thamilselvan and J. G. R. Sathiaseelan "Detection and Classification of Lung Cancer MRI Images by using Enhanced K Nearest Neighbor Algorithm" Indian Journal of Science and Technology, Vol 9(43) Nov 2016.

\section{Authors Profile}

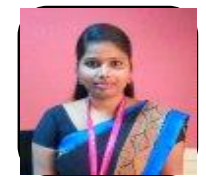

P Kavitha, Assistant Professor, Department of Information Technology, Bharath Institute of Higher Education and Research.

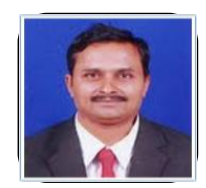

S.Prabakaran, Professor, Department of Computer Science and Engineering, SRM University 